\title{
ON THE GIBBS PHENOMENON FOR HARMONIC MEANS
}

\author{
FU CHENG HSIANG
}

1. Let a sequence of functions $\left\{f_{n}(x)\right\}$ converge to a function $f(x)$ for $x_{0}<x \leqq x_{0}+h$, say. If, for $n$ and $1 /\left(x-x_{0}\right)$ tending to $+\infty$ independently of each other, $\lim \sup f_{n}(x)>f\left(x_{0}+0\right)$, or if $\lim \inf f_{n}(x)$ $<f\left(x_{0}+0\right)$, then we say that the sequence $\left\{f_{n}(x)\right\}$ possesses the Gibbs phenomenon in the right hand neighborhood of the point $x=x_{0}$. A similar definition holds for the lefthand neighborhood. Let $f(x)$ be an arbitrary function having a simple discontinuity at the point $x_{0}$. The function

$$
g(x)=f(x)-\frac{l\left(x_{0}\right)}{\pi} \sum_{\nu=1}^{\infty} \frac{\sin \nu\left(x-x_{0}\right)}{\nu},
$$

where $l\left(x_{0}\right)=f\left(x_{0}+0\right)-f\left(x_{0}-0\right)$, is continuous at $x_{0}$. If, in addition, $f(x)$ is of bounded variation in a neighborhood of $x_{0}$, since the Fourier series of $g(x)$ converges uniformly at the point $x_{0}$, the behavior of the Fourier series of $f(x)^{1}$ near the point $x_{0}$ can thus be dominated by the behavior of the series

$$
\frac{l\left(x_{0}\right)}{\pi} \sum_{\nu=1}^{\infty} \frac{\sin \nu\left(x-x_{0}\right)}{\nu}
$$

and accordingly the Gibbs phenomenon of $f(x)$ near the simple discontinuity $x_{0}$ can be indicated by the study of the special series

$$
\frac{\pi-x}{2} \sim \sum_{\nu=1}^{\infty} \frac{\sin \nu x}{\nu}
$$

near the point $x=0[6, \S 8.51$, p. 181$]$. Furthermore, let $\left(\rho_{n}\right)$ be a sequence of numbers. If

$$
\frac{1}{\log n} \sum_{\nu=1}^{n} \frac{\rho_{n-\nu}}{\nu} \rightarrow s
$$

as $n \rightarrow \infty$, then we say that $\left(\rho_{n}\right)$ is summable by harmonic means to the sum $s$. It is known that if $\left(\rho_{n}\right)$ converges to $s$, then it is also summable by harmonic means to the same sum.

The Gibbs phenomenon for the Cesàro, Euler, Hausdorff and Borel means have been studied exhaustively by Cramér, Gronwall, Szász

Received by the editors March 4, 1959 and, in revised form, May 5, 1959.

${ }^{1}$ Of course, we take here $f_{n}(x)=\left(l\left(x_{0}\right) / \pi\right) \sum_{\nu=1}^{n}(1 / \nu) \sin \nu x$. 
and Lorch $[1 ; 2 ; 3 ; 4 ; 5]$. In this note, we shall show that the Gibbs phenomenon for the harmonic means is present at the simple discontinuity $x_{0}$ of the function $f(x)$ which is of bounded variation in the neighborhood of $x_{0}$. Our result is the following

THEOREM. The Gibbs phenomenon for the harmonic means of the sequence

$$
d_{n}(x)=\sum_{\nu=1}^{n} \frac{\sin \nu x}{\nu}
$$

presents itself at the point $x=0$ and has the Gibbs ratio

$$
\frac{2}{\pi} \int_{0}^{\pi} \frac{\sin t}{t} d t
$$

2. We have

$$
\begin{aligned}
d_{n}(x) & =\sum_{v=1}^{n} \int_{0}^{x} \cos v t d t \\
& =\int_{0}^{x} \frac{\sin (n+1 / 2) t}{2 \sin (t / 2)} d t+O(x) \\
& =\int_{0}^{x} \frac{\sin n t}{t} d t+O\left(\frac{1}{n}\right)+O(x) \\
& =\int_{x / n}^{x} \frac{\sin n t}{t} d t+O\left(\frac{1}{n}\right)+O(x) .
\end{aligned}
$$

Denote

$$
\tau_{n}(x)=\frac{1}{\log n} \sum_{\nu=1}^{n} \frac{d_{n-\nu}(x)}{\nu} .
$$

Then

$$
\begin{aligned}
\tau_{n}(x)= & \frac{1}{\log n} \int_{x / n}^{x} \frac{1}{t} \sum_{\nu=1}^{n} \frac{\sin (n-\nu) t}{\nu} d t+O\left(\frac{1}{n}\right)+O(x) \\
= & \frac{1}{\log n} \int_{x / n}^{x} \frac{1}{t} \sum_{\nu=1}^{n} \frac{1}{\nu}(\sin n t \cos \nu t-\cos n t \sin \nu t) d t \\
& +O\left(\frac{1}{n}\right)+O(x) \\
= & \frac{1}{\log n}\left(I_{1}-I_{2}\right)+O\left(\frac{1}{n}\right)+O(x)
\end{aligned}
$$

say. Write 


$$
I_{1}=\int_{x / n}^{x} \frac{\sin n t}{t}\left(\int_{t}^{0}\left(\sum_{\nu=1}^{n} \sin \nu v\right) d v\right) d t+\left(\sum_{\nu=1}^{n} \frac{1}{\nu}\right) \int_{x / n}^{x} \frac{\sin n t}{t} d t .
$$

Then,

$$
\begin{aligned}
I_{1}= & \int_{x / n}^{x} \frac{\sin n t}{t}\left(\int_{0}^{t} \frac{\cos (n+1 / 2) v-\cos (v / 2)}{2 \sin (v / 2)} d v\right) d t \\
& +\left(\sum_{v=1}^{n} \frac{1}{\nu}\right) \int_{x / n}^{x} \frac{\sin n t}{t} d t \\
= & \int_{x / n}^{x} \frac{\sin n t}{t}\left(\int_{0}^{t} \frac{\sin ^{2}(n v / 2)}{\tan (v / 2)} d v\right) d t \\
& -\frac{1}{2} \int_{x / n}^{x} \frac{\sin n t}{t}\left(\int_{0}^{t} \sin n v d v\right) d t+\left(\sum_{\nu=1}^{n} \frac{1}{\nu}\right) \int_{x / n}^{x} \frac{\sin n t}{t} d t \\
= & 2 \int_{x / n}^{x} \frac{\sin n t}{t}\left(\int_{0}^{t} \frac{\sin ^{2}(n v / 2)}{v} d v\right) d t \\
& -\frac{1}{2} \int_{x / n}^{x} \frac{\sin n t}{t}\left(\int_{0}^{t} \sin n v d v\right) d t+\left(\sum_{\nu=1}^{n} \frac{1}{v}\right) \int_{x / n}^{x} \frac{\sin n t}{t} d t \\
& +O(x),
\end{aligned}
$$

since the function $h(v)=1 / v-1 /(2 \tan (v / 2))$ is bounded, so that

$$
\begin{gathered}
\int_{x / n}^{x} \frac{\sin n t}{t}\left(\int_{0}^{t} \sin ^{2}(n v / 2)\left(\frac{1}{v}-\frac{1}{2 \tan (v / 2)}\right) d v\right) d t \\
=O\left(\int_{x / n}^{x}|\sin n t| d t\right)=O(x) .
\end{gathered}
$$

By reversing the order of integration, we find

$$
\begin{aligned}
I_{1}= & 2 \int_{0}^{x / n} \frac{\sin ^{2}(n v / 2)}{v}\left(\int_{x / n}^{x} \frac{\sin n t}{t} d t\right) d v \\
& +2 \int_{x / n}^{x} \frac{\sin ^{2}(n v / 2)}{v}\left(\int_{v}^{x} \frac{\sin n t}{t} d t\right) d v \\
& +\left(\sum_{\nu=1}^{n} \frac{1}{\nu}\right) \int_{x / n}^{x} \frac{\sin n t}{t} d t+O(x)+O\left(\frac{1}{n}\right) \\
= & I_{3}+I_{4}+\left(\sum_{\nu=1}^{n} \frac{1}{\nu}\right) \int_{x / n}^{x} \frac{\sin n t}{t} d t+O(x)+O\left(\frac{1}{n}\right),
\end{aligned}
$$


say.

$$
\begin{aligned}
\left|I_{3}\right| & \leqq 2\left|\int_{x / n}^{x} \frac{\sin n t}{t} d t\right| \cdot \int_{0}^{x / n} \frac{\sin ^{2}(n v / 2)}{v} d v \\
& =O\left(\int_{0}^{x / n} \frac{\sin ^{2}(n v / 2)}{v} d v\right) \\
& =O\left(\int_{0}^{x / n} \frac{|\sin (n v / 2)|}{v} d v\right) \\
& =O(x) .
\end{aligned}
$$

Furthermore, if $x=l / n, l>0$ being an arbitrary constant and to be given a definite value later, then

$$
\begin{aligned}
\left|I_{4}\right| & \leqq 2 \int_{0}^{n x} \frac{\sin ^{2}(t / 2)}{t}\left|\int_{t}^{n x} \frac{\sin u}{u} d u\right| d t \\
& =2 \int_{0}^{t} \frac{\sin ^{2}(t / 2)}{t}\left|\int_{t}^{l} \frac{\sin u}{u} d u\right| d t \\
& =O\left(\int_{0}^{l} \frac{\sin ^{2}(t / 2)}{t} d t\right) \\
& =O(1) .
\end{aligned}
$$

Moreover,

$$
\begin{aligned}
I_{2}= & \int_{x / n}^{x} \frac{\cos n t}{t}\left(\int_{0}^{t}\left(\sum_{v=1}^{n} \cos \nu v\right) d v\right) d t \\
= & \int_{x / n}^{x} \frac{\cos n t}{t}\left(\int_{0}^{t} \frac{\sin (n+1 / 2) v}{2 \sin (v / 2)} d v\right) d t+o\left(\frac{1}{n}\right) \\
= & \int_{x / n}^{x} \frac{\cos n t}{t}\left(\int_{0}^{t} \frac{\sin n v}{2 \tan (v / 2)} d v\right) d t \\
& +\frac{1}{2} \int_{x / n}^{x} \frac{\cos n t}{t}\left(\int_{0}^{t} \cos n v d v\right) d t+O\left(\frac{1}{n}\right) \\
= & \int_{x / n}^{x} \frac{\cos n t}{t}\left(\int_{0}^{t} \frac{\sin n v}{2 \tan (v / 2)} d v\right) d t+O\left(\frac{1}{n}\right) \\
= & \int_{x / n}^{x} \frac{\cos n t}{t}\left(\int_{0}^{t} \frac{\sin n v}{v} d v\right) d t+O\left(\frac{\log n}{n}\right) \\
= & I_{5}+O\left(\frac{\log n}{n}\right),
\end{aligned}
$$


since

$$
\begin{aligned}
\mid \int_{x / n}^{x} \frac{\cos n t}{t}\left(\int _ { 0 } ^ { t } \left(\frac{1}{v}\right.\right. & \left.\left.-\frac{1}{2 \tan (v / 2)}\right) \sin n v d v\right) d t \mid \\
& \leqq \int_{x / n}^{x} \frac{1}{t}\left|\int_{0}^{t}\left(\frac{1}{v}-\frac{1}{2 \tan (v / 2)}\right) \sin n v d v\right| d t \\
& \leqq \frac{A}{n} \int_{x / n}^{x} \frac{d t}{t} \\
& =O\left(\frac{\log n}{n}\right) .
\end{aligned}
$$

Now,

$$
\begin{aligned}
& I_{5}=\int_{0}^{x / n} \frac{\sin n v}{v}\left(\int_{x / n}^{x} \frac{\cos n t}{t} d t\right) d v+\int_{x / n}^{x} \frac{\sin n v}{v}\left(\int_{0}^{x} \frac{\cos n t}{t} d t\right) d v \\
& =\int_{0}^{x / n} \frac{\sin n v}{v}\left(\int_{x / n}^{x} \frac{d t}{t}\right) d v-2 \int_{0}^{x / n} \frac{\sin n v}{v}\left(\int_{x / n}^{x} \frac{\sin ^{2}(n t / 2)}{t} d t\right) d v \\
& +\int_{x / n}^{x} \frac{\sin n v}{v} \log \frac{x}{v} d v-2 \int_{0}^{x / n} \frac{\sin n v}{v}\left(\int_{0}^{x} \frac{\sin ^{2}(n t / 2)}{t} d t\right) d v \\
& =\int_{x / n}^{x} \frac{\sin n v}{v} \log \frac{x}{v} d v-2 \int_{0}^{x / n} \frac{\sin n v}{v}\left(\int_{v}^{x} \frac{\sin ^{2}(n t / 2)}{t} d t\right) d v \\
& +O(x \log n) \\
& =I_{6}-2 I_{7}+O(1) \text {. } \\
& \left|I_{7}\right| \leqq \int_{0}^{x / n} \frac{|\sin n v|}{v}\left(\int_{0}^{x} \frac{\sin ^{2}(n t / 2)}{t} d t\right) d v \\
& =\int_{0}^{x / n} \frac{|\sin n v|}{v} d v \int_{0}^{x} \frac{\sin ^{2}(n t / 2)}{t} d t \\
& =O\left(n^{2} x^{2} \int_{0}^{x / n} \frac{|\sin n v|}{v} d v\right) \\
& =O\left(n^{2} x^{3}\right) \\
& =O\left(\frac{1}{n}\right) \text {, }
\end{aligned}
$$

if $l=n x$ as previously assigned. Making the transformation $n v=u$, we see that 


$$
\begin{aligned}
I_{6} & =\int_{x}^{n x} \frac{\sin u}{u} \log \frac{n x}{u} d u \\
& =\int_{l / n}^{l} \frac{\sin u}{u} \log \frac{l}{u} d u
\end{aligned}
$$

where $l=n x$ as given before. Thus,

$$
\begin{aligned}
I_{6} & =O(1)+\int_{l / n}^{l} \frac{\sin u}{u} \log \frac{1}{u} d u \\
& =O(1)+O\left(\int_{0}^{1} \log \frac{1}{u} d u\right) \\
& =O(1) .
\end{aligned}
$$

3. From the above analysis, it follows that

$$
\tau_{n}(x)=\frac{1}{\log n}\left(\sum_{\nu=1}^{n} \frac{1}{\nu}\right) \int_{x / n}^{x} \frac{\sin n t}{t} d t+O\left(\frac{x}{\log n}\right)+O\left(\frac{1}{\log n}\right) .
$$

Let $x=l / n, l>0$ as assigned previously. Then, we obtain

$$
\begin{aligned}
\tau_{n}\left(\frac{l}{n}\right) & =\int_{0}^{l / n} \frac{\sin n t}{t} d t+o\left(\frac{1}{\log n}\right) \\
& =\int_{0}^{l} \frac{\sin t}{t} d t+O\left(\frac{1}{\log n}\right) .
\end{aligned}
$$

In particular, taking $l=\pi$, we obtain the upper limit of the sequence $\left\{\tau_{n}(x)\right\}$ as $n$ and $1 / x$ tend to infinity independently of each other. E.g.,

$$
\limsup _{x \rightarrow 0, n \rightarrow \infty} \tau_{n}(x)=\int_{0}^{\pi} \frac{\sin t}{t} d t .
$$

This displays that the Gibbs phenomenon for the harmonic means of sequence of functions $\left\{d_{n}(x)\right\}$ presents itself at the point $x=0$ and has the Gibbs ratio as indicated in $\$ 1$. The theorem is thus completely established.

\section{REFERENCES}

1. H. Cramér, Etudes sur la sommation des séries de Fourier, Ark. Mat. Astronomi och Fysik vol. 13 (1919) pp. 1-21.

2. T. H. Gronwall, Zur Gibbs'schen Erscheinung, Ann. of Math. (2) vol. 31 (1930) pp. 233-240.

3. L. Lorch, The Gibbs phenomenon for Borel means, Proc. Amer. Math. Soc. vol. 9 (1957) pp. 81-84. 
4. O. Szász, On the Gibbs phenomenon for Euler means, Acta Sci. Math. Szeged Part B vol. 12 (1950) pp. 107-111.

5. - , Gibbs phenomenon for Hausdorff means, Trans. Amer. Math. Soc. vol. 69 (1950) pp. 440-456.

6. A. Zygmund, Trigonometrical series, Warsaw, 1935.

National Taiwan University, Taiwan, China

\section{A NEW CLASS OF INTEGRAL TRANSFORMS}

\section{TA $L I^{1}$}

Introduction. In aerodynamics, a velocity potential is usually represented as a singular integral given by a sink and source distribution. The integrand contains a strength factor. For cones and slender bodies, this strength factor is determined by methods $[1]^{2}$ either graphical or numerical as suggested by Tsien [2], von Kármán and Moore [3], and other writers. An exact solution was believed to be too difficult, for an integral equation of the first kind does not, in general, admit a solution.

In deriving a solution of a certain aerodynamical problem, the writer was led to a much more general class of integral equations, each of which has, as its kernel, a Chebyshev polynomial of the first kind, divided by the square root of the difference of two squares. The writer was fortunate in having found the exact solution to each of these singular integral equations. The solution is given in the form of a singular integral involving a Chebyshev polynomial of the first kind. The application of these results to aerodynamics is immediate.

This paper is dedicated to Professor O. Perron, of Munich, on his 79th birthday, in appreciation of his achievement as a mathematician and for his success as a teacher.

Chebyshev polynomials of the first kind. The Chebyshev polynomial of the first kind and $n$th degree is denoted by $T_{n}(t)$. It is defined as the polynomial solution of the differential equation

$$
\left(x^{2}-1\right) y^{\prime \prime}(x)+x y^{\prime}(x)-n^{2} y(x)=0
$$

Presented to the Society, January 21, 1959; received by the editors March 30, 1959 and, in revised form, May 4, 1959 and May 25, 1959.

${ }^{1}$ Design Specialist, Dynamics Group, Convair-Astronautics, Convair Division of General Dynamics Corporation, San Diego, California; Lecturer, University of California, Los Angeles, California.

${ }^{2}$ Numbers in brackets indicate references at the end of this paper. 